\title{
Paclitaxel for treatment of advanced small cell lung cancer (SCLC): a retrospective study of 185 patients
}

\author{
Damian von Eiff ${ }^{1,2 \#}$, Farastuk Bozorgmehr ${ }^{1,2 \#}$, Inn Chung ${ }^{1,2}$, Denise Bernhardt ${ }^{3,4}$, Stefan Rieken ${ }^{3,4}$, \\ Stephan Liersch $^{5}$, Thomas Muley ${ }^{2,6}$, Sonja Kobinger ${ }^{6}$, Michael Thomas ${ }^{1,2}$, Petros Christopoulos ${ }^{1,2 *}$, \\ Martin Steins ${ }^{1,2 *}$
}

${ }^{1}$ Department of Thoracic Oncology, Thoraxklinik at Heidelberg University Hospital, Heidelberg, Germany; ${ }^{2}$ Translational Lung Research Center Heidelberg (TLRC-H), Member of the German Center for Lung Research (DZL), Germany; ${ }^{3}$ Heidelberg University Hospital, Department of Radiation Oncology, Heidelberg, Germany; ${ }^{4}$ Heidelberg Institute of Radiation Oncology (HIRO), Heidelberg, Germany; ${ }^{5}$ Pharmacy Department, Thoraxklinik at Heidelberg University Hospital, Heidelberg, Germany; ${ }^{6}$ Translational Research Unit, Thoraxklinik at Heidelberg University Hospital, Heidelberg, Germany

Contributions: (I) Conception and design: D von Eiff, F Bozorgmehr, M Thomas, P Christopoulos, M Steins; (II) Administrative support: None; (III) Provision of study materials or patients: None; (IV) Collection and assembly of data: D von Eiff, F Bozorgmehr, I Chung, D Bernhardt, S Rieken, S Liersch, T Muley, S Kobinger; (V) Data analysis and interpretation: D von Eiff, F Bozorgmehr, I Chung, P Christopoulos; (VI) Manuscript writing: All authors; (VII) Final approval of manuscript: All authors.

"These authors contributed equally to this work.

*Both co-senior authors contributed equally to this work.

Correspondence to: Martin Steins, MD. Thoraxklinik at Heidelberg University Hospital, Röntgenstr. 1, 69126 Heidelberg, Germany.

Email: Martin.Steins@med.uni-heidelberg.de.

Background: Etoposide-/platinum-based chemotherapy is the standard first-line treatment for extensivedisease small cell lung cancer (SCLC), but responses are short-lived and subsequent options limited. Here, we present our experience with paclitaxel in advanced treatment lines.

Methods: We retrospectively studied the clinical course of all paclitaxel-treated SCLC patients between 2005 and 2015 in our institution. Prognostic and predictive factors were analyzed by Kaplan-Meier and Cox regression analyses.

Results: A total of 185 patients [119 men, median age 65 years, median ECOG performance status (PS) 1] were identified. One hundred and sixty-eight patients had extensive disease (ED) at the time of paclitaxel therapy. Paclitaxel was mainly given as third- or fourth-line therapy (93\%). The response rate (RR) was $17 \%$ and disease control rate (DCR) 28\%. Patients reached a median progression-free survival (PFS) of 1.6 (95\% CI: 1.4-1.8) months and median overall survival (OS) of 3.3 (95\% CI: 2.8-3.9) months. Main toxicities were fatigue $(25 \%)$ and polyneuropathy $(17 \%)$. Dose reduction of $\geq 25 \%$ was associated with shorter PFS [1.9 (95\% CI: $1.5-2.3$ ) vs. 1.4 (95\% CI: 1.3-1.5) months; $\mathrm{P}=0.004]$. Further independent predictive factors for PFS were gender, age, and hepatic/brain metastases $(\mathrm{P}<0.05)$. Tumor response to paclitaxel, PS, number and location of metastases, dose reduction, and smoking history were significant factors for OS in univariable analyses $(\mathrm{P}<0.05)$, while PS, dose reduction, status of cerebral/hepatic metastases, tumor response, and smoking history were retained as independent prognostic factors in multivariable testing. Notably, ECOG PS 2 patients had toxicity rates similar to ECOG PS $0-1$ patients (63\% vs. 62\%), as well as a comparable DCR (29\% vs. 28\%), which was associated with prolonged survival (4.5 vs. 3.2 months for refractory cases, $\mathrm{P}=0.034)$.

Conclusions: Paclitaxel has clinically relevant activity in heavily pretreated SCLC. While patients with good PS and no cerebral/hepatic metastases derive the greatest benefit, ECOG PS 2 per se should not be used as a criterion to exclude patients.

Keywords: Small cell lung cancer (SCLC); paclitaxel; progressive disease (PD) 
Submitted Jul 22, 2019. Accepted for publication Nov 19, 2019.

doi: $10.21037 /$ jtd.2019.12.74

View this article at: http://dx.doi.org/10.21037/jtd.2019.12.74

\section{Introduction}

Small cell lung cancer (SCLC) accounts for $12-15 \%$ of all lung cancer cases worldwide and is among the most common causes of cancer-related mortality. SCLC is an aggressive tumor that frequently develops widespread metastases early, resulting in a dismal prognosis with a median overall survival (OS) of $<10$ months in patients with extensive disease (ED) and a 5 -year OS rate of $1-5 \%$ (1). First-line treatment for ED-SCLC patients usually comprises a combination of etoposide and platinum-based chemotherapy, which has a high overall response rate (ORR) of up to $70 \%$ (1). However, the rapid development of chemoresistance in SCLC tumors will inevitably cause relapse, and alternative systemic therapies have to be exploited. For second-line treatment, topotecan monotherapy has been approved after a randomized phase III trial showed a survival benefit compared with best supportive care (2). In addition, drugs like vincristine, doxorubicin, cyclophosphamide, paclitaxel, amrubicin, gemcitabine and trofosfamide in monotherapy or in combination regimens can be administered upon progression.

About $20 \%$ to $30 \%$ of SCLC patients are treated with a third-line therapy, and approximately one-third of these patients will proceed to a fourth line of systemic treatment (3-9). However, information on prognostic factors and the efficacies of different treatments in such advanced therapy lines is scarce. Clinical trial phase III data are not available for patients who have undergone three or more lines of therapy. Moreover, only few retrospective studies have addressed the efficacy of chemotherapeutic drugs in advanced treatment lines, analyzing less than 40 patients in third or higher chemotherapy lines (4,7-14), with some exceptions $(3,6,15)$. These studies investigated several chemotherapeutic regimens, again decreasing the number of patients analyzed with respect to individual therapies. Several reports identified prognostic factors for progressionfree survival (PFS) or OS of SCLC patients in advanced lines of chemotherapy, mainly in third-line chemotherapy. A significant prognostic value of the patient's performance status (PS) was observed in different studies $(5,6,15)$. Furthermore, the type and duration of tumor response to second-line therapy appears to be prognostic for outcome of third-line treatment (6-8). A prognostic value has also been attributed to factors such as liver metastasis, body mass index as well as levels of lactate dehydrogenase, C-reactive protein and hemoglobin $(5,7,8,15)$.

Paclitaxel belongs to the family of taxanes and interferes with cell division by stabilizing microtubules $(16,17)$. In SCLC patients, it is widely used in single-agent or in combination therapy. Frontline paclitaxel monotherapy achieves response rates (RRs) ranging from $33 \%$ to $53 \%$ (18-20). Two phase II trials reported RRs between 24 and $29 \%$ with paclitaxel monotherapy in pretreated SCLC $(21,22)$. A recent retrospective analysis reported a RR of $24 \%$ in 34 patients who received paclitaxel in monotherapy or in combination with platinum agents as third line (5).

Here, we report our observations in 185 heavily pretreated SCLC patients who received paclitaxel monotherapy in our institution and discuss implementations into clinical practice to aid in this difficult therapeutic situation.

\section{Methods}

\section{Patient selection and clinical data}

In this retrospective study, we evaluated SCLC patients who received paclitaxel monotherapy in our institution between 2005 and 2015. Patients were excluded in case of histologically and/or cytologically ambiguously classifiable SCLC, especially mixed bronchial tumors, simultaneous secondary tumors, or malignant tumors within 5 years prior to SCLC diagnosis. Patients were also excluded from the analysis if treatment was carried out in other hospitals and diagnostic or therapeutic courses were not fully available (Figure 1). Clinical data were collected through a review of patient records.

The following patient data were collected: gender, age, ECOG (Eastern Cooperative Oncology Group) PS, number of pack years, and date of death. Tumor characteristics included stage at initial diagnosis and at start of paclitaxel therapy, as well as number and location of metastases at paclitaxel initiation. Tumor stage was classified as limited disease (LD) and ED according to the classification of the 


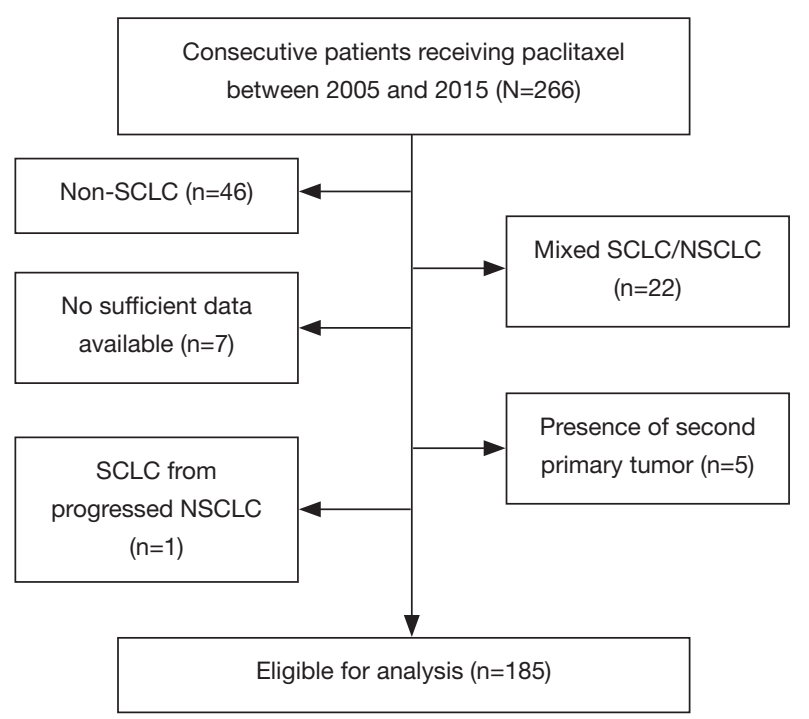

Figure 1 Patient selection criteria of patients included in this study. 220 patients treated with paclitaxel at Thoraxklinik at Heidelberg University Hospital between 2005 and 2015 were retrospectively reviewed. In total, 35 patients were excluded either due to mixed histology, insufficient data availability or presence of another primary tumor. 185 patients were eligible for analysis. SCLC, small cell lung cancer.

Veterans Administration Lung Study Group (23).

Regarding paclitaxel therapy, the following parameters were included: number of cycles, start and stop date, number of line in which paclitaxel was given, dose reductions, and dose delays.

Toxicities documented in the medical records during and after paclitaxel medication were assessed using the Common Terminology Criteria for Adverse Events (CTCAE, version 5.0).

\section{Evaluation of response and survival}

Response to paclitaxel was evaluated according to response evaluation criteria in solid tumors (RECIST) with crosssectional imaging studies every 6 to 8 weeks (24). In case of clinical signs of progression, scans were performed earlier.

PFS was defined as the time from the first dose of paclitaxel to documented disease progression or death, whichever occurred first. If the date of disease progression was unknown, PFS was calculated using the date of death or last follow-up visit and patients were censored. A similar analysis was conducted to determine OS.

Time to progression (TTP) was defined as interval between the last day of previous chemotherapy just before paclitaxel and the day of disease progression.

\section{Statistical analysis}

The effect of quantitative (e.g., gender) and continuous (age and pack years) parameters on survival (PFS, OS) was analyzed according to Kaplan Meier with a log-rank test or using Cox regression, respectively. Variables with significant effect in univariable testing were then also included in multivariable Cox regression analyses for OS and PFS. Categorical variables were compared with a Chi-Square test. Statistical analysis was conducted using the SPSS Statistics 24 software (IBM Corporation, New York, USA).

\section{Results}

\section{Patient baseline characteristics}

Among 220 patients treated with paclitaxel in our institution between 2005 and 2015, we identified 185 patients eligible for analysis according to the above-mentioned criteria. Figure 1 shows the exclusion criteria of the 35 patients that were not analyzed; relevant patient characteristics are summarized in Table 1.

The median age of patients at initiation of paclitaxel therapy was 65 years (range, 38-87 years). About two-thirds of the patients were men (119 cases, 64\%). Sixty-six percent of patients had ECOG PS 1, 21\% had ECOG PS 2, and $14 \%$ ECOG PS 0 . The majority of patients had a history of smoking (93\%), of which $42 \%$ were active smokers at the time of SCLC diagnosis. About half of the patients had initially been diagnosed with LD (49\%), but at time of paclitaxel initiation, the proportion of patients with ED had increased to $91 \%$. Sixty-three percent of these patients had metastases in more than one organ. The distribution of metastases in brain, liver and other sites is shown in Table 1 . Patients had been treated with at least two and up to seven lines of chemotherapy (four lines of therapy in average). The majority of them had received platinum-based chemotherapy in the first line (94\%), followed by topotecan in the second line $(81 \%)$. One hundred seventy-three patients (94\%) received third-line therapy, and 128 patients $(69 \%)$ a fourth line of treatment. Thirty-six patients (19\%) received more than four lines of therapy. An overview of 
Table 1 Patient characteristics $(\mathrm{N}=185)$

\begin{tabular}{|c|c|c|}
\hline Variable & $\mathrm{n}$ & $\%$ \\
\hline \multicolumn{3}{|l|}{ Sex } \\
\hline Male & 119 & 64 \\
\hline Female & 66 & 36 \\
\hline \multicolumn{3}{|l|}{ Age } \\
\hline$<65$ years & 87 & 47 \\
\hline$\geq 65$ years & 98 & 53 \\
\hline \multicolumn{3}{|l|}{ ECOG } \\
\hline 0 & 25 & 13.5 \\
\hline 1 & 122 & 66 \\
\hline 2 & 38 & 20.5 \\
\hline \multicolumn{3}{|l|}{ Stage of disease at diagnosis } \\
\hline LD & 90 & 49 \\
\hline ED & 95 & 51 \\
\hline \multicolumn{3}{|l|}{ Number of metastatic sites at start of paclitaxel therapy } \\
\hline No metastases & 17 & 9 \\
\hline 1 organ system & 52 & 28 \\
\hline$>1$ organ system & 116 & 63 \\
\hline \multicolumn{3}{|l|}{ Metastases according to location } \\
\hline Non-hepatic/non-cerebral & 44 & 24 \\
\hline $\begin{array}{l}\text { Hepatic metastases [hepatic only and hepatic and } \\
\text { other organ (except brain)] }\end{array}$ & 68 & 37 \\
\hline $\begin{array}{l}\text { Cerebral metastases [cerebral only and cerebral } \\
\text { and other organ (except liver)] }\end{array}$ & 25 & 13.5 \\
\hline Hepatic and cerebral metastases & 31 & 17 \\
\hline \multicolumn{3}{|l|}{ Smoking history } \\
\hline Unknown & 11 & 6 \\
\hline No & 2 & 1.1 \\
\hline Yes & 172 & 93 \\
\hline Ongoing & 78 & 42 \\
\hline$<6$ months cessation & 35 & 19 \\
\hline$>6$ months cessation & 57 & 31 \\
\hline Unknown & 2 & 1 \\
\hline
\end{tabular}

therapy lines is shown in Table 2 .

\section{Paclitaxel: time and dose of drug delivery}

Paclitaxel was mainly given as a fourth-line (48\%) or thirdline therapy (33\%). Time points of paclitaxel therapy are shown in Table 3.

Of 185 patients, $134(72 \%)$ received paclitaxel at the dose of $175 \mathrm{mg} / \mathrm{m}^{2}$ every three weeks. Paclitaxel was dosed as recommended by the NCCN guidelines, based on a previous trial indicating a survival benefit in SCLC patients (21). Dose reductions were required in 51 patients: in 22 patients (12\%), the paclitaxel dose was reduced by less than $25 \%$, in 29 patients (16\%), by $25 \%$ and more.

The majority of patients with paclitaxel dose adjustment (46 patients, 90\%) received the reduced dose already in the first cycle due to adverse events in previous lines, mostly severe hematotoxicity (in 28 cases, $61 \%$ ). Moreover, 4 patients ( $8 \%$ ) had previously experienced cardiac problems and in 5 patients $(11 \%)$ the paclitaxel dose was adjusted prophylactically, as the patients' general condition had significantly deteriorated under previous therapy. Other reasons for dose modifications are listed in Table 3.

\section{Toxicity}

Side effects documented in the medical records during and after paclitaxel medication were predominantly grade 1 or 2 . Toxicities and grading are displayed for all patients in Table 4 and stratified according to ECOG PS in Table S1. Adverse events of any grade occurred in $58 \%$ of patients, grade $3 / 4$ events were documented in $15 \%$ of patients. The most frequent complication after paclitaxel administration was fatigue in 46 patients ( $25 \%$ of cases for all grades; $5 \%$ for grades 3 and 4). The most frequent grade $3 / 4$ adverse event was hematotoxicity (9\%), predominantly with leukocytopenia grade 3/4 in 7 cases (4\%). Polyneuropathy of all grades was newly diagnosed in 32 of 185 patients (17\%), 3 patients $(1.5 \%)$ had a grade $3 / 4$ polyneuropathy. Twenty-two patients (12\%) reported polyneuropathy from previous cytostatic therapy lines, in three of which neuropathy increased during paclitaxel treatment. 
Table 2 Lines of chemotherapy*

\begin{tabular}{|c|c|c|c|c|c|c|}
\hline Chemotherapy regimen & $\begin{array}{c}\text { 1st line } \\
(n=185 ; 100 \%)\end{array}$ & $\begin{array}{c}\text { 2nd line } \\
(n=185 ; 100 \%)\end{array}$ & $\begin{array}{c}\text { 3rd line } \\
(n=173 ; 94 \%)\end{array}$ & $\begin{array}{c}\text { 4th line } \\
(n=134 ; 72 \%)\end{array}$ & $\begin{array}{c}\text { 5th line } \\
(n=66 ; 36 \%)\end{array}$ & $\begin{array}{l}\text { 6th line and beyond } \\
\qquad(\mathrm{n}=22 ; 12 \%)\end{array}$ \\
\hline Platinum/etoposide & 174 & 19 & 1 & 7 & 10 & 2 \\
\hline Clinical trial & 2 & & & & & \\
\hline Paclitaxel & & 12 & 61 & 89 & 22 & $2^{\#}$ \\
\hline Amrubicin & & 5 & & & & \\
\hline Vincristine & & 1 & & & & \\
\hline Doxorubicin & & & & & 1 & \\
\hline Gemcitabine & & & & & 1 & 2 \\
\hline Etoposide & & & & & & 1 \\
\hline
\end{tabular}

*, re-challenges with the same chemotherapy regimen were considered a subsequent line of therapy; \#, paclitaxel in combination with carboplatin (this case was not included in paclitaxel analysis).

Arthralgia and myalgia were reported in 28 patients ( $15 \%$ for all grades, $1.5 \%$ grade $3 / 4)$. Twenty-five patients $(13.5 \%$ for all grades; $3 \%$ grade 3/4) experienced nausea and vomiting. Allergic reactions with exanthema were documented in 6 patients $(3 \%), 1$ of them $(0.5 \%)$ with a grade $3 / 4$ reaction. An increase in serum transaminases exceeding twice the upper normal limit was observed in 7 patients ( $4 \%$ for all grades; $1 \%$ grade $3 / 4)$. No fatal toxicities were documented.

No toxicities were documented in 11 cases $(6 \%)$, as patients had been transferred to external hospitals or palliative wards due to deterioration of their general condition or disease progression.

Toxicity rates were comparable in patients with ECOG PS $0-1$ and ECOG PS 2 (Table S1). In rare cases, slight numerical differences were observed, such as $4 \%$ vs. $9 \%$ for ECOG PS 0-1 vs. ECOG PS 2 for fatigue grade 3/4, but these were not statistically significant $(\mathrm{P}=0.31$ with a Chisquare test).

\section{Efficacy of paclitaxel treatment}

In total, therapeutic response could be assessed in 132 (71\%) of the 185 paclitaxel-treated patients. Thirty-one patients $(17 \%)$ achieved partial remission (PR) as "best response" to paclitaxel therapy, irrespectively of the therapy line (Table 5). In addition, 21 patients (11\%) had stable disease (SD), resulting in a disease control rate (DCR) of $28 \%$. A discordant tumor response to paclitaxel was observed in 8 patients (4\%). 39\% of patients not respond to paclitaxel therapy, i.e., had progressive disease (PD). Complete remission was not observed.

The response appears to depend on the applied paclitaxel dose (Table S2), as patients receiving the full dose $(\mathrm{n}=134$; $72 \%$ of all patients) achieved PR in 25 cases (19\%) and SD in 18 cases $(13 \%)$, reflecting a DCR of $32 \%$. In contrast, among the patients with paclitaxel dose reductions, the DCR was only $18 \%$, with PR in 6 patients (12\%) and SD in 3 patients (6\%). Thirty-seven percent of these patients did not respond at all (PD) and in another 37\%, staging was not possible.

Tumor response was independent of the therapy line of paclitaxel treatment (Table 5), which was most frequently the third $(\mathrm{n}=61)$ or fourth line $(\mathrm{n}=89)$. In these therapy lines, PR and disease control were observed in about $15 \%$ and $25 \%$ of patients, respectively. Similarly, paclitaxel treatment in the second $(n=12)$ and fifth plus sixth therapy line $(n=23)$ 
Table 3 Characteristics of paclitaxel therapy $(\mathrm{N}=185)$

\begin{tabular}{|c|c|c|}
\hline Variable & $\mathrm{n}$ & $\%$ \\
\hline \multicolumn{3}{|l|}{ Therapy line } \\
\hline 1 & 0 & 0 \\
\hline 2 & 12 & 6.5 \\
\hline 3 & 61 & 33 \\
\hline 4 & 89 & 48 \\
\hline 5 & 22 & 12 \\
\hline 6 & 1 & 0.5 \\
\hline \multicolumn{3}{|l|}{ Dose reduction } \\
\hline No dose reduction & 134 & 72 \\
\hline$<25 \%$ & 22 & 12 \\
\hline$\geq 25 \%$ & 29 & 16 \\
\hline \multicolumn{3}{|l|}{ Time point of dose reduction $(n=51)$} \\
\hline First cycle & 44 & 86 \\
\hline Second cycle & 4 & 8 \\
\hline Third cycle & 1 & 2 \\
\hline $\begin{array}{l}\text { Repeated dose reductions beginning } \\
\text { with 1st cycle }\end{array}$ & 1 & 2 \\
\hline Only first cycle & 1 & 2 \\
\hline \multicolumn{3}{|l|}{$\begin{array}{l}\text { Reasons for dose reductions starting with } \\
\text { first cycle (referring to therapy lines before } \\
\text { paclitaxel, } n=46 \text { ) }\end{array}$} \\
\hline Hematotoxicity & 28 & 61 \\
\hline Reduced general condition & 5 & 11 \\
\hline Cardiac comorbidities & 4 & 9 \\
\hline Bad tolerance of previous chemotherapy & 3 & 7 \\
\hline Polyneuropathy & 2 & 4 \\
\hline Concomitant radiotherapy & 2 & 4 \\
\hline Unknown & 2 & 4 \\
\hline
\end{tabular}

resulted in PR in $25 \%$ and $22 \%$ of patients, respectively (Table 5).

Median TTP just before paclitaxel therapy was 22.5 days for all paclitaxel-treated patients. There was no correlation between TTP and disease control (TTP 50 vs. 34 days for patients achieving disease control or not, $\mathrm{P}=0.10$ with a $t$-test).

In 53 patients (29\%), tumor response was not radiologically evaluated because the patients' clinical condition deteriorated and/or they died prematurely,
Table 4 Toxicities during paclitaxel therapy

\begin{tabular}{lcc}
\hline Toxicities & All grades, $\mathrm{n}[\%]$ & CTC grade 3/4, n [\%] \\
\hline Any toxicity & $108[58]$ & $28[15]$ \\
Fatigue & $46[25]$ & $9[5]$ \\
Polyneuropathy & $32[17]$ & $3[1.5]$ \\
Arthralgia/myalgia & $28[15]$ & $3[1.5]$ \\
Any hematotoxicity & $27[14.5]$ & $11[6]$ \\
Leukocytopenia & $12[6.5]$ & $7[4]$ \\
Anemia & $11[6]$ & $1[0.5]$ \\
Thrombocytopenia & $5[3]$ & $2[1]$ \\
Pancytopenia & $2[1]$ & $1[0.5]$ \\
Nausea/vomiting & $25[13.5]$ & $6[3]$ \\
Hepatotoxicity & $7[4]$ & $2[1]$ \\
Allergic reaction & $6[3]$ & $1[0.5]$ \\
\hline
\end{tabular}

*, multiple entries for one patient possible.

Table 5 Efficacy of paclitaxel therapy

\begin{tabular}{lccccc}
\hline $\begin{array}{l}\text { Best response, } \\
\mathrm{n}[\%]\end{array}$ & $\begin{array}{c}\text { 2nd line } \\
(\mathrm{n}=12)\end{array}$ & $\begin{array}{c}\text { 3rd line } \\
(\mathrm{n}=61)\end{array}$ & $\begin{array}{c}4 \text { th line } \\
(\mathrm{n}=89)\end{array}$ & $\begin{array}{c}5 \text { th and } \\
\text { 6th line } \\
(\mathrm{n}=23)\end{array}$ & $\begin{array}{c}\text { Overall } \\
(\mathrm{n}=185)\end{array}$ \\
\hline $\mathrm{DCR}$ & $6[50]$ & $15[25]$ & $24[27]$ & $7[31]$ & $52[28]$ \\
$\mathrm{PR}$ & $3[25]$ & $9[15]$ & $14[16]$ & $5[22]$ & $31[17]$ \\
$\mathrm{SD}$ & $3[25]$ & $6[10]$ & $10[11]$ & $2[9]$ & $21[11]$ \\
$\mathrm{PD}$ & 0 & $24[39]$ & $38[43]$ & $10[43]$ & $72[39]$ \\
Discordant & 0 & $4[7]$ & $4[4]$ & 0 & $8[4]$ \\
$\begin{array}{l}\text { Unknown } \\
\text { (no restaging }\end{array}$ & $6[50]$ & $18[29]$ & $23[26]$ & $6[26]$ & $53[29]$ \\
performed ${ }^{*}$ & & & & \\
\hline
\end{tabular}

precluding further imaging studies. These patients were not included in response evaluation. Overrepresented among them were cases with ECOG PS 2 (21/38 of ECOP PS 2 cases vs. 32/147 ECOG PS $0-1$ cases, $\mathrm{P}<0.01$ ), while age and timing of paclitaxel therapy were not associated with clinical deterioration leading to omission of restaging. Metastatic status was only associated with clinical deterioration in case of both hepatic and cerebral metastases in patients with ECOG PS $2(\mathrm{P}=0.011)$. However, the entire patient subset with ECOG PS 2 showed a DCR of 29\% (Table S3), which was comparable with the DCR of $28 \%$ observed in the entire 

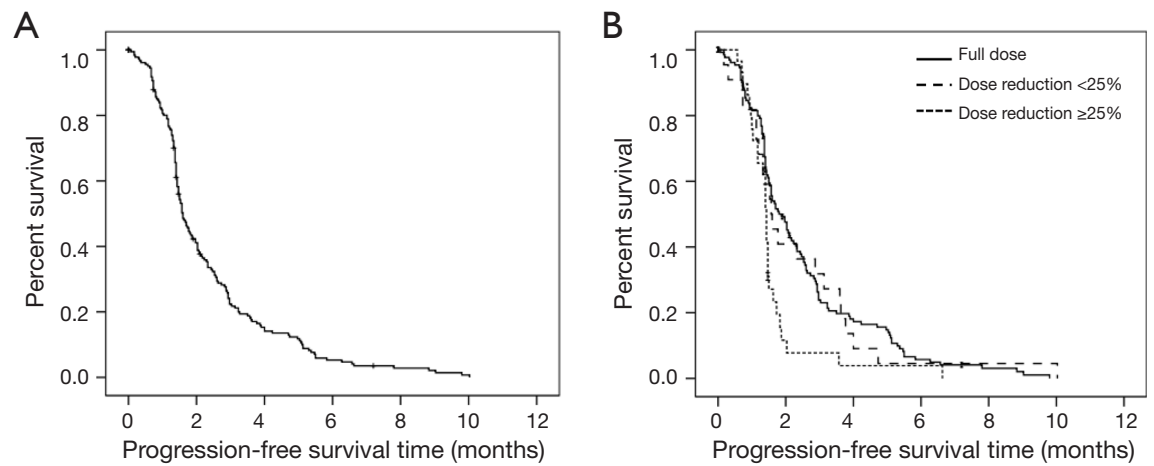

Figure 2 Progression-free survival (PFS) of paclitaxel-treated patients. (A) Kaplan Meier PFS analysis of all paclitaxel-treated SCLC patients ( $\mathrm{n}=185$, median PFS =1.6 months); (B) stratification according to administered paclitaxel dose: median PFS =1.9 vs. 1.6 vs. 1.4 months for full dose $v$ s. dose reduction $<25 \%$ vs. dose reduction $\geq 25 \%$; $\log$ rank-test for trend $\mathrm{P}=0.013$ ).

cohort (Table 5). A detailed response pattern in patients with ECOG PS 2 is displayed in Table S3.

\section{Predictive and prognostic factors for paclitaxel therapy}

All patients were followed-up for at least 12 months. Median PFS was 1.6 months (95\% CI: 1.4-1.8; range 0.1-10.0) (Figure $2 A$ ). In univariate analysis, dose reduction of $\geq 25 \%$ was associated with a significantly shorter median PFS compared to the recommended paclitaxel dose [1.4 (95\% CI: 1.3-1.5) vs. 1.9 (1.5-2.3) months; $\mathrm{P}=0.004]$ (Figure 2B). There was no significant $(\mathrm{P}=0.12)$ association between gender and PFS, however, significantly more female patients received a reduced paclitaxel dose (38\% females vs. $22 \%$ males, $\mathrm{P}=0.021$ with a Chi-Square test), which itself was an unfavorable factor and expected to obscure a potentially beneficial effect of gender per se. Age was also predictive for PFS (HR 0.98, 95\% CI: 0.96-0.998, P=0.03), while pack years, PS and timing of paclitaxel therapy were not associated with PFS. Also, the number of metastatic sites did not affect PFS, however, the location of metastases did have a statistically significant impact on PFS $(\mathrm{P}=0.03)$, as without hepatic and cerebral metastases, the median PFS was 2.2 (95\% CI: 1.5-2.9) months, while in case of both cerebral and hepatic metastases, it was only 1.5 (1.1-1.9) months. Gender, age, dose reduction, and presence of both hepatic and cerebral metastases were retained as independent predictive factors for PFS in a multivariable analysis (Table 6).

Median OS was 3.3 months (95\% CI: 2.8-3.9; range 0.125.7) (Figure $3 A$ ). Significant factors for OS obtained from univariate analysis were PS [ECOG PS $0-1 v s$. ECOG PS
2, 3.8 (95\% CI: 3.0-4.6) vs. 2.5 (1.4-3.5) months; $\mathrm{P}=0.002]$ (Figure 3B), number of metastatic sites [no metastatic sites $v s$. metastases in 1 organ system $v s$. metastases in $>1$ organ systems; 5.7 (95\% CI 4.5-6.8) vs. 4.4 (3.0-5.9) vs. 3.1 (2.73.6) months; $\mathrm{P}=0.038$ ] (Figure $3 C$ ), presence of cerebral and/or hepatic metastases [non-cerebral/non-hepatic 4.7 months (95\% CI: 3.4-6.1) vs. hepatic 3.3 months (2.24.4) $(\mathrm{P}=0.008)$ vs. cerebral 3.3 months (2.6-4.0) $(\mathrm{P}=0.047)$ vs. both 2.1 months $(1.1-3.1)(\mathrm{P}<0.001)]$ (Figure 3D). Other metastatic sites did not have a significant impact on survival (data not shown). Since patients with hepatic and cerebral metastases appeared to have a similar outcome, they were grouped together in subsequent analyses.

Further prognostic factors for OS were dose reduction [no dose reduction $v s$. dose reduction $\geq 25 \%, 3.9$ (95\% CI: 2.9-4.8) vs. 2.5 (1.6-3.3) months, $\mathrm{P}=0.004$ ] (Figure 3E), response [DC vs. $\mathrm{PD} /$ discordant response $v s$. no staging, 6.4 (95\% CI: 5.4-7.4) vs. 3.8 (3.0-4.6) vs. 1.5 (1.1-1.9) months; $\mathrm{P}<0.001$ ] (Figure S1A), and the degree of tobacco exposure (pack years) (HR 1.007, 95\% CI: 1.001-1.013, $\mathrm{P}=0.025)$. Response as a prognostic factor was further analyzed according to ECOG PS (Figure $S 1 B, C$ ). Since patients with ECOG PS $0-1$ represent the majority of the study population, results regarding this sub-population were comparable to those of the entire population [DC vs. $\mathrm{PD} /$ discordant response vs. no staging, 7 (95\% CI: 5.8-8.2) vs. 3.9 (3.1-4.7) vs. 1.7 (1.4-1.9) months; $\mathrm{P}<0.001]$ (Figure $S 1 B$ ). As expected, survival rates were lower in patients with ECOG PS 2 [DC $v s$. PD/discordant response $v s$. no staging, 4.5 (95\% CI: 3.9-5.1) vs. 3.2 (2.3-4.2) vs. $0.9(0.7-1.2)$ months; $\mathrm{P}<0.001]$, however, response to paclitaxel treatment significantly increased 
Table 6 Results of multivariate Cox regression analysis

\begin{tabular}{|c|c|c|c|c|}
\hline Variable & $\mathrm{N}^{*} \mathrm{H}$ & $\mathrm{HR}(\operatorname{Exp}(\mathrm{B}))^{\star \star}$ & ${ }^{*} 95 \% \mathrm{Cl}^{\star \star \star}$ & Significance \\
\hline \multicolumn{5}{|l|}{ PFS } \\
\hline \multicolumn{5}{|l|}{ Gender } \\
\hline Male & 110 & & & \\
\hline Female & 58 & 0.653 & $0.5-0.9$ & 0.018 \\
\hline \multicolumn{5}{|l|}{ Dose reduction } \\
\hline No dose reduction & 120 & & & \\
\hline$<25 \%$ & 21 & 1.160 & $07-1.9$ & 0.552 \\
\hline$\geq 25 \%$ & 27 & 2.550 & $1.6-4.1$ & $<0.001$ \\
\hline Age & 165 & 0.968 & $0.95-0.99$ & 0.002 \\
\hline \multicolumn{5}{|c|}{ Localization of metastatic sites } \\
\hline $\begin{array}{l}\text { Non-hepatic/non- } \\
\text { cerebral }\end{array}$ & 44 & & & \\
\hline Hepatic or cerebral & 93 & 1.120 & $0.7-1.7$ & 0.575 \\
\hline Hepatic and cerebral & 31 & 1.652 & $1.0-2.7$ & 0.045 \\
\hline \multicolumn{5}{|l|}{ os } \\
\hline \multicolumn{5}{|l|}{$\begin{array}{l}\text { ECOG performance- } \\
\text { status }\end{array}$} \\
\hline ECOG $0-1$ & 117 & & & \\
\hline ECOG 2 & 35 & 1.751 & $1.1-2.7$ & 0.012 \\
\hline \multicolumn{5}{|c|}{ Number of metastatic sites } \\
\hline \multicolumn{5}{|c|}{ One metastatic site 48} \\
\hline$\geq 2$ metastatic sites & 104 & 0.947 & $0.6-1.4$ & 0.802 \\
\hline \multicolumn{5}{|c|}{ Localization of metastatic sites } \\
\hline $\begin{array}{l}\text { Non-hepatic/non- } \\
\text { cerebral }\end{array}$ & 41 & & & \\
\hline Hepatic or cerebral & 85 & 1.886 & $1.2-2.9$ & 0.004 \\
\hline $\begin{array}{l}\text { Hepatic and } \\
\text { cerebral }\end{array}$ & 26 & 2.712 & $1.5-5.0$ & 0.001 \\
\hline \multicolumn{5}{|l|}{ Dose reduction } \\
\hline No dose reduction & 109 & & & \\
\hline$<25 \%$ & 18 & 0.733 & $0.4-1.3$ & 0.313 \\
\hline$\geq 25 \%$ & 25 & 1.762 & $1.1-2.9$ & 0.024 \\
\hline \multicolumn{5}{|l|}{ Tumor response } \\
\hline \multicolumn{5}{|c|}{ No staging possible 47} \\
\hline PD/discordant & 64 & 0.154 & $0.1-0.3$ & $<0.001$ \\
\hline Disease control & 41 & 0.069 & $0.04-0.1$ & $<0.001$ \\
\hline Pack years & 150 & 1.751 & $1.00-1.02$ & 0.012 \\
\hline
\end{tabular}

*, patients included in this analysis; ${ }^{\star \star}$, hazard ratio; ${ }^{\star \star \star}, 95 \%$ confidence-interval.
OS also in this especially fragile population (DC $v s$. PD/ discordant, 4.5 vs. 3.2 months: $\mathrm{P}=0.034$ ) (Figure S1C). Gender, age and timing of paclitaxel therapy (Figure $3 F$ ) were not associated with OS.

PS, presence of cerebral and hepatic metastases, dose reduction $\geq 25 \%$, response to paclitaxel therapy and pack years were identified as independent prognostic factors for OS in a multivariable analysis (Table 6).

Overall, patients that achieved disease control under paclitaxel treatment (28\%) had a substantial survival benefit with a PFS gain of 2.6 months [4 (95\% CI: 2.7-5.3) vs. 1.4 (1.36-1.44) months; $\mathrm{P}<0.001]$ and an OS gain of 3.7 months [6.4 (95\% CI: 5.4-7.4) vs. 2.7 (2.3-3.1) months, $\mathrm{P} \leq 0.001$ ], compared to the rest of patients.

\section{Discussion}

Several studies have retrospectively investigated cohorts of SCLC patients with respect to prognostic factors of firstand second-line therapy, and a few reports have analyzed more advanced treatment lines. However, since there is a lack of clinical standards for progressed SCLC, it is crucial to gain more insight into efficacies and prognostic factors of systemic therapies for advanced treatment of recurrent disease in a real-life setting.

Here, clinical data of 185 SCLC patients who received single-agent paclitaxel therapy were analyzed retrospectively. To our knowledge, this is the largest number of patients reported in a retrospective analysis of paclitaxel therapy in SCLC. Most patients received paclitaxel as a thirdor fourth-line therapy. Taking into account all patients, paclitaxel monotherapy resulted in a DCR of $28 \%$, with $17 \%$ of patients displaying PR and $11 \%$ SD. In the patient population that was treated with the full dose of paclitaxel ( $n=130)$, a DCR of $32 \%$ was observed. The median PFS starting from paclitaxel initiation was 1.6 months and the median OS was 3.3 months. Paclitaxel responders had a longer median OS (6.4 months) compared to patients with paclitaxel-resistant tumors (3.8 months). Prospective phase II studies investigating paclitaxel therapy in SCLC patients receiving second- and third-line treatment have reported RRs between $24 \%$ and $29 \%$ and an OS from 3.3 to 5.8 months; a retrospective analysis with 40 patients receiving paclitaxel as third-line therapy described an RR of $23.5 \%$ and an OS of 5.9 months $(5,21,22)$. Compared to these studies, the population in our analysis had lower RRs and OS. Since this study is based on a retrospective analysis 

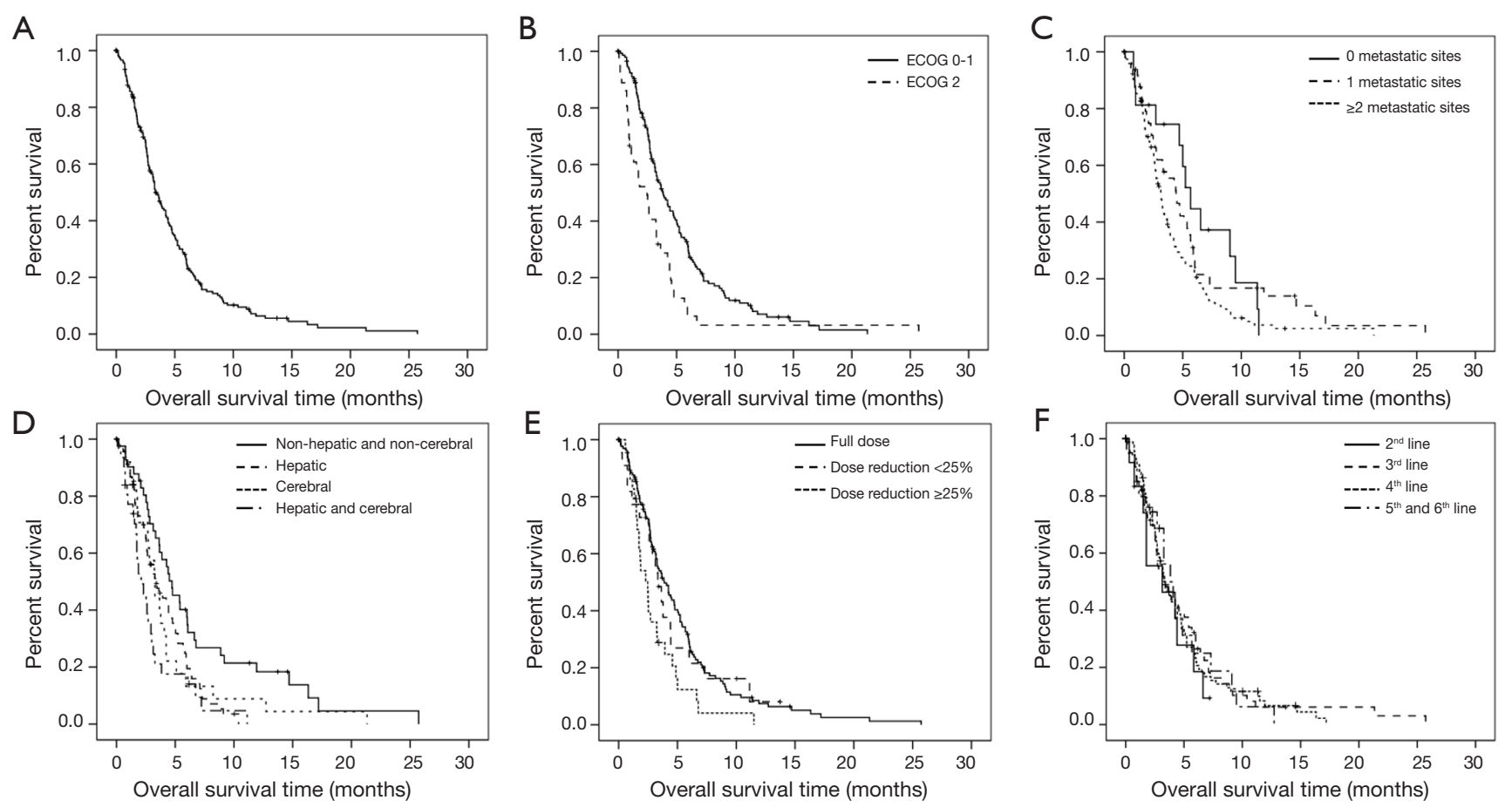

Figure 3 Overall survival (OS) of paclitaxel-treated patients. (A) Kaplan Meier OS analysis of all paclitaxel-treated patients in this study ( $\mathrm{n}=185$, median OS =3.3 months); (B) stratification according to ECOG performance status (3.8 vs. 2.5 months for ECOG PS 0-1 vs. ECOG PS 2; $\mathrm{P}=0.002 ;(\mathrm{C})$ stratification according to number of metastatic sites $(5.7$ vs. 4.4 vs. 3.1 months for no metastatic sites vs. metastases in 1 organ system vs. metastases in $>1$ organ system; $\mathrm{P}=0.038$ ); (D) presence of cerebral and/or hepatic metastases [4.7 vs. 3.3 vs. 3.3 vs. 2.1 months for non-cerebral/non-hepatic vs. hepatic $(\mathrm{P}=0.008)$ vs. cerebral $(\mathrm{P}=0.047)$ vs. both $(\mathrm{P}<0.001)]$; (E) stratification according to administered paclitaxel dose (3.9 vs. 2.5 months for full dose $v s$. dose reduction $\geq 25 \%$; $=0.004$ ); and (F) stratification according to timing of paclitaxel therapy (3.1 vs. 3.4 vs. 3.3 vs. 3.8 months for 2 nd-line vs. 3 rd-line vs. 4th-line vs. 5th-line and 6th-line; $\mathrm{P}=0.86$ ).

of real-world data, it is likely that fewer patients were excluded from paclitaxel therapy compared with highly preselected populations enrolled in prospective trials.

In $29 \%$ of the patients, tumor response could not be evaluated radiologically due to the patients' clinical condition and/or premature death. While there was a significant correlation between ECOG PS 2 and clinical deterioration, there was no correlation between disease control and ECOG PS. No significant association could be detected between TTP and disease control. Since disease control itself is significantly associated with OS of paclitaxel-treated patients (Figure S1A), TTP should not be used for excluding pretreated patients for paclitaxel therapy. Thirty percent of our patients did not receive paclitaxel at the full dose, which was associated with a decline in PFS and OS (Figures 2B,3E). There was no significant correlation between ECOG PS and PFS, suggesting that the impaired outcome is not due to a generally poorer condition of patients with paclitaxel dose reduction. Instead, dose reductions were mainly determined by the high occurrence of severe hematotoxicities in previous therapy lines, which is particularly important given that paclitaxel is applied in rather advanced lines of SCLC therapy. Considering these observations, a reduction in paclitaxel dose should be avoided if possible, as intensification of supportive therapy (e.g., granulocyte-colony stimulating factor) may enable paclitaxel therapy at full dose.

In line with previous reports, we found that survival rates were significantly lower in SCLC patients with distant metastases in multiple organs systems in univariate analysis $(\mathrm{P}=0.038)$ (Figure 3C) $(25,26)$. With respect to PFS, however, our analysis did not reveal any association with the number of metastatic sites, suggesting that patients with a high tumor burden still benefit from paclitaxel treatment in terms of disease control. We further analyzed the localization of metastases and found an independent prognostic 
value for the presence of hepatic and cerebral metastases, as has been described before (Figure 3D) (25,27-29). Non-hepatic/non-cerebral metastases were associated with longer OS compared to hepatic or cerebral metastases, and presence of both hepatic and cerebral metastases resulted in the worst survival rate. In addition, ECOG PS 2 was independently associated with a significant reduction in OS, similar to what has been described before (Figure 3B) $(6,9,30,31)$. However, DCR was not lower in case of ECOG PS 2 in our paclitaxel-treated patients: the DCR was $29 \%$ in patients with ECOG PS 2 and 28\% in the entire cohort. In addition, the achievement of disease control under paclitaxel therapy was associated with a significantly prolonged OS also within the subset of ECOG PS 2 patients (4.5 vs. 3.2 months from the start of paclitaxel treatment, $\mathrm{P}=0.039$, Figure S1C), regardless of the fact that ECOG PS 2 patients had an overall shorter survival than ECOG PS 0-1 patients (2.5 vs. 3.8 months $\mathrm{P}=0.002$; Figure $3 B$ ). Furthermore, toxicity was not higher in ECOG PS 2 patients: $63 \% v s$. $62 \%$ for any toxicity of any grade and $14 \%$ vs. $16.5 \%$ for any toxicity of grade 3/4 in ECOG PS 2 vs. 0-1 patients, respectively (Table S1). Therefore, we believe that ECOG PS 2 per se should not be used as a criterion for exclusion of patients from paclitaxel therapy. However, our results suggest that ECOG PS 2 patients with concomitant brain and liver metastases should not be offered paclitaxel, since this was frequently followed by rapid clinical deterioration.

Interestingly, the number of pack years also affected survival significantly and independently in this cohort with an increase in mortality by $1 \%$ with every additional pack year $(\operatorname{Exp}(B)=1.013)$. The prognostic value of smoking has been described before $(32,33)$, however, not in a population in later therapy lines. Hence, even in patients with very advanced disease, the number of smoked cigarettes still had an impact on OS. This should be emphasized when approaching patients regarding smoking cessation.

A major limitation of this study is its retrospective single-center nature, because a bias in patient selection cannot be excluded. However, the fact that PS, metastatic status, dose reduction, tumor response and pack years showed prognostic value in our analysis similar to other published series $(6,9,25-33)$, suggests that the analyzed cohort represents a typical SCLC patient population. While several studies have retrospectively analyzed the effect of second- and third-line SCLC therapy, partially in small sets of patients $(4,5,7,10,11,34)$, there are only two studies that describe RRs of SCLC therapy beyond the third line. These studies included relatively few cases [27 patients (3) and 73 patients (35)] and additionally grouped outcomes across different substances. To our knowledge, no data are available on RRs beyond a third line of therapy for a specific substance, including paclitaxel. Studying a relatively large cohort, we were able to describe RRs to paclitaxel in comparatively large patient numbers in $4^{\text {th }}$ line (89 patients) and $5^{\text {th }}$ line plus $6^{\text {th }}$ line (23 patients) therapy with a DCR of $27 \%$ and $31 \%$, respectively (Tables 3,5). Therefore, our results provide insights into the clinical use of paclitaxel and confirm a reasonable efficiency of this therapy with a DCR of $28 \%$, even in heavily pretreated patients with advanced SCLC. Notably, the observed RRs of $15-23 \%$ for paclitaxel in third and higher therapy lines were similar or slightly better compared to that reported for the immune checkpoint inhibitor nivolumab (12\%) (36). Thus, despite the rapid emergence of novel therapeutic possibilities, paclitaxel monotherapy should still be considered as a reasonable option for the treatment of progressed SCLC patients. Particularly patients in a very good or good general condition and absence of liver and brain metastases benefit from this generally well-tolerated treatment. However, paclitaxel therapy in patients with ECOG PS 2 is still justified, as our results show a clinically relevant DCR of $29 \%$ associated with prolonged survival as well as lack of excessive toxicity in this fragile population. Based on our observation that dose reductions result in shorter survival, we strongly recommend a critical assessment of the expected toxicity before considering such measures and emphasize the use of maximal supportive therapy to reduce side effects.

In conclusion, our results highlight the value of paclitaxel monotherapy in heavily pretreated, advanced SCLC, a very difficult therapeutic situation still lacking clinical standards.

\section{Acknowledgments}

Funding: We acknowledge financial support by Deutsche Forschungsgemeinschaft within the funding programme Open Access Publishing, by the Baden-Württemberg Ministry of Science, Research and the Arts and by Ruprecht-Karls-Universität Heidelberg.

\section{Footnote}

Conflicts of Interest: PC serves as the unpaid editorial board member of Fournal of Thoracic Disease from Dec 2018 to Nov 2020. The authors report the following relevant financial activities unrelated to the submitted work: F 
Bozorgmehr has received research funding from BMS, Astra Zeneca and honoraria from Novartis, MSD. S Rieken reports honoraria and travel expenses from Elekta Inc., Accuray Inc., AstraZeneca, BMS, Lilly, Roche, DGP, DKG, Degro; IITs and other research funding from BMS, Accuray Inc., Merck KGaA; Consulting or advisory role: Accuray Inc., AstraZeneca GmbH. S Liersch reports advisory board honoraria from BMS, Amgen, MSD and speaker's honoraria from MSD, Sanofi. T Muley reports research grants, travel expenses, honorarium for report and patent applications from Roche and research grants from Chugai Pharma. M Thomas reports advisory board honoraria from AbbVie, BMS, Boehringer, Celgene, Lilly, MSD, Novartis, Roche, Takeda; speaker's honoraria from Lilly, MSD, Takeda; research funding from AstraZeneca, BMS, Celgene, Roche and travel grants from BMS, Boehringer, MSD, Novartis. PC reports lecture fees from Roche, Chugai and Novartis. The other authors have no conflicts of interest to declare.

Ethical Statement: The authors are accountable for all aspects of the work in ensuring that questions related to the accuracy or integrity of any part of the work are appropriately investigated and resolved. This study was approved by the Ethics committee of the Heidelberg University (S-461/2016) and was conducted in accordance with the Declaration of Helsinki as revised in 2013. Written informed consent was obtained from all patients prior to treatment and anonymity preserved at all times.

Open Access Statement: This is an Open Access article distributed in accordance with the Creative Commons Attribution-NonCommercial-NoDerivs 4.0 International License (CC BY-NC-ND 4.0), which permits the noncommercial replication and distribution of the article with the strict proviso that no changes or edits are made and the original work is properly cited (including links to both the formal publication through the relevant DOI and the license). See: https://creativecommons.org/licenses/by-nc-nd/4.0/.

\section{References}

1. Sabari JK, Lok BH, Laird JH, et al. Unravelling the biology of SCLC: implications for therapy. Nat Rev Clin Oncol 2017;14:549-61.

2. O'Brien ME, Ciuleanu TE, Tsekov H, et al. Phase III trial comparing supportive care alone with supportive care with oral topotecan in patients with relapsed small-cell lung cancer. J Clin Oncol 2006;24:5441-7.
3. Nagy-Mignotte H, Guillem P, Vignoud L, et al. Outcomes in recurrent small-cell lung cancer after one to four chemotherapy lines: a retrospective study of 300 patients. Lung Cancer 2012;78:112-20.

4. de Jong WK, ten Hacken NH, Groen HJ. Third-line chemotherapy for small cell lung cancer. Lung Cancer 2006;52:339-42.

5. Kim SH, Kim MJ, Kim YJ, et al. Paclitaxel as thirdline chemotherapy for small cell lung cancer failing both etoposide- and camptothecin-based chemotherapy. Medicine (Baltimore) 2017;96:e8176.

6. Saruwatari K, Umemura S, Nomura S, et al. Prognostic Factor Analysis in Patients With Small-Cell Lung Cancer Treated With Third-Line Chemotherapy. Clin Lung Cancer 2016;17:581-7.

7. Simos D, Sajjady G, Sergi M, et al. Third-line chemotherapy in small-cell lung cancer: an international analysis. Clin Lung Cancer 2014;15:110-8.

8. Inomata M, Hayashi R, Tokui K, et al. Outcome and prognostic factors in patients with small cell lung cancer who receive third-line chemotherapy. Tumori 2014;100:507-11.

9. Minami $S$, Ogata $Y$, Ihara $S$, et al. Retrospective analysis of outcomes and prognostic factors of chemotherapy for small-cell lung cancer. Lung Cancer (Auckl) 2016;7:35-44.

10. Park S, Ahn MJ, Ahn JS, et al. Combination chemotherapy with paclitaxel and ifosfamide as the third-line regimen in patients with heavily pretreated small cell lung cancer. Lung Cancer 2007;58:116-22.

11. Lebeau B, Chouaid C, Baud M, et al. Oral secondand third-line lomustine-etoposide-cyclophosphamide chemotherapy for small cell lung cancer. Lung Cancer 2010;67:188-93.

12. Jacot W, Pujol JL, Chakra M, et al. Epirubicin and ifosfamide in relapsed or refractory small cell lung cancer patients. Lung Cancer 2012;75:213-6.

13. Genestreti G, Tiseo M, Kenmotsu H, et al. Outcomes of Platinum-Sensitive Small-Cell Lung Cancer Patients Treated With Platinum/Etoposide Rechallenge: A MultiInstitutional Retrospective Analysis. Clin Lung Cancer 2015;16:e223-8.

14. Aktas G, Kus T, Kalender ME, et al. Survival analysis in second-line and third-line chemotherapy with irinotecan followed by topotecan or topotecan followed by irinotecan for extensive-stage small-cell lung cancer patients: a single-center retrospective study. Onco Targets Ther 2016;9:1921-6.

15. Fiegl M, Pircher A, Waldthaler C, et al. Small steps of 
improvement in small-cell lung cancer (SCLC) within two decades: a comprehensive analysis of 484 patients. Lung Cancer 2014;84:168-74.

16. Barbuti AM, Chen ZS. Paclitaxel Through the Ages of Anticancer Therapy: Exploring Its Role in Chemoresistance and Radiation Therapy. Cancers (Basel) 2015;7:2360-71.

17. de Weger VA, Beijnen JH, Schellens JH. Cellular and clinical pharmacology of the taxanes docetaxel and paclitaxel--a review. Anticancer Drugs 2014;25:488-94.

18. Ettinger DS, Finkelstein DM, Sarma RP, et al. Phase II study of paclitaxel in patients with extensive-disease smallcell lung cancer: an Eastern Cooperative Oncology Group study. J Clin Oncol 1995;13:1430-5.

19. Graziano SL, Herndon JE 2nd, Socinski MA, et al. Phase II trial of weekly dose-dense paclitaxel in extensive-stage small cell lung cancer: cancer and leukemia group B study 39901. J Thorac Oncol 2008;3:158-62.

20. Kirschling RJ, Grill JP, Marks RS, et al. Paclitaxel and G-CSF in previously untreated patients with extensive stage small-cell lung cancer: a phase II study of the North Central Cancer Treatment Group. Am J Clin Oncol 1999;22:517-22.

21. Smit EF, Fokkema E, Biesma B, et al. A phase II study of paclitaxel in heavily pretreated patients with small-cell lung cancer. Br J Cancer 1998;77:347-51.

22. Yamamoto N, Tsurutani J, Yoshimura N, et al. Phase II study of weekly paclitaxel for relapsed and refractory small cell lung cancer. Anticancer Res 2006;26:777-81.

23. Zelen M. Keynote address on biostatistics and data retrieval. Cancer Chemother Rep 3 1973;4:31-42.

24. Eisenhauer EA, Therasse P, Bogaerts J, et al. New response evaluation criteria in solid tumours: revised RECIST guideline (version 1.1). Eur J Cancer 2009;45:228-47.

25. Ren Y, Dai C, Zheng H, et al. Prognostic effect of liver metastasis in lung cancer patients with distant metastasis. Oncotarget 2016;7:53245-53.

26. Nakazawa K, Kurishima K, Tamura T, et al. Specific organ metastases and survival in small cell lung cancer. Oncol

Cite this article as: von Eiff D, Bozorgmehr F, Chung I, Bernhardt D, Rieken S, Liersch S, Muley T, Kobinger S, Thomas M, Christopoulos P, Steins M. Paclitaxel for treatment of advanced small cell lung cancer (SCLC): a retrospective study of 185 patients. J Thorac Dis 2020;12(3):782-793. doi: $10.21037 /$ jtd.2019.12.74
Lett 2012;4:617-20.

27. Christodolou C, Pavlidis N, Samantas E, et al. Prognostic factors in Greek patients with small cell lung cancer (SCLC). A Hellenic Cooperative Oncology Group study. Anticancer Res 2002;22:3749-57.

28. Brueckl WM, Herbst L, Lechler A, et al. Predictive and prognostic factors in small cell lung carcinoma (SCLC)-analysis from routine clinical practice. Anticancer Res 2006;26:4825-32.

29. Ogino H, Hanibuchi M, Kakiuchi S, et al. Analysis of the Prognostic Factors of Extensive Disease Small-Cell Lung Cancer Patients in Tokushima University Hospital. J Med Invest 2016;63:286-93.

30. Korkmaz T, Seber S, Kefeli U, et al. Comparison of second-line treatment outcomes between sensitive and refractory small cell lung cancer patients: a retrospective analysis. Clin Transl Oncol 2013;15:535-40.

31. Spiegelman D, Maurer LH, Ware JH, et al. Prognostic factors in small-cell carcinoma of the lung: an analysis of 1,521 patients. J Clin Oncol 1989;7:344-54.

32. Ou SH, Ziogas A, Zell JA. Prognostic factors for survival in extensive stage small cell lung cancer (ED-SCLC): the importance of smoking history, socioeconomic and marital statuses, and ethnicity. J Thorac Oncol 2009;4:37-43.

33. Chen J, Jiang R, Garces YI, et al. Prognostic factors for limited-stage small cell lung cancer: a study of 284 patients. Lung Cancer 2010;67:221-6.

34. Hagmann R, Hess V, Zippelius A, et al. SecondLine Therapy of Small-Cell Lung Cancer: Topotecan Compared to a Combination Treatment with Adriamycin, Cyclophosphamide And Vincristine (ACO) - a Single Center Experience. J Cancer 2015;6:1148-54.

35. Coutinho AD, Shah M, Lunacsek OE, et al. Real-world treatment patterns and outcomes of patients with small cell lung cancer progressing after 2 lines of therapy. Lung Cancer 2019;127:53-8.

36. Ready N, Farago AF, de Braud F, et al. Third-Line Nivolumab Monotherapy in Recurrent SCLC: CheckMate 032. J Thorac Oncol 2019;14:237-44. 

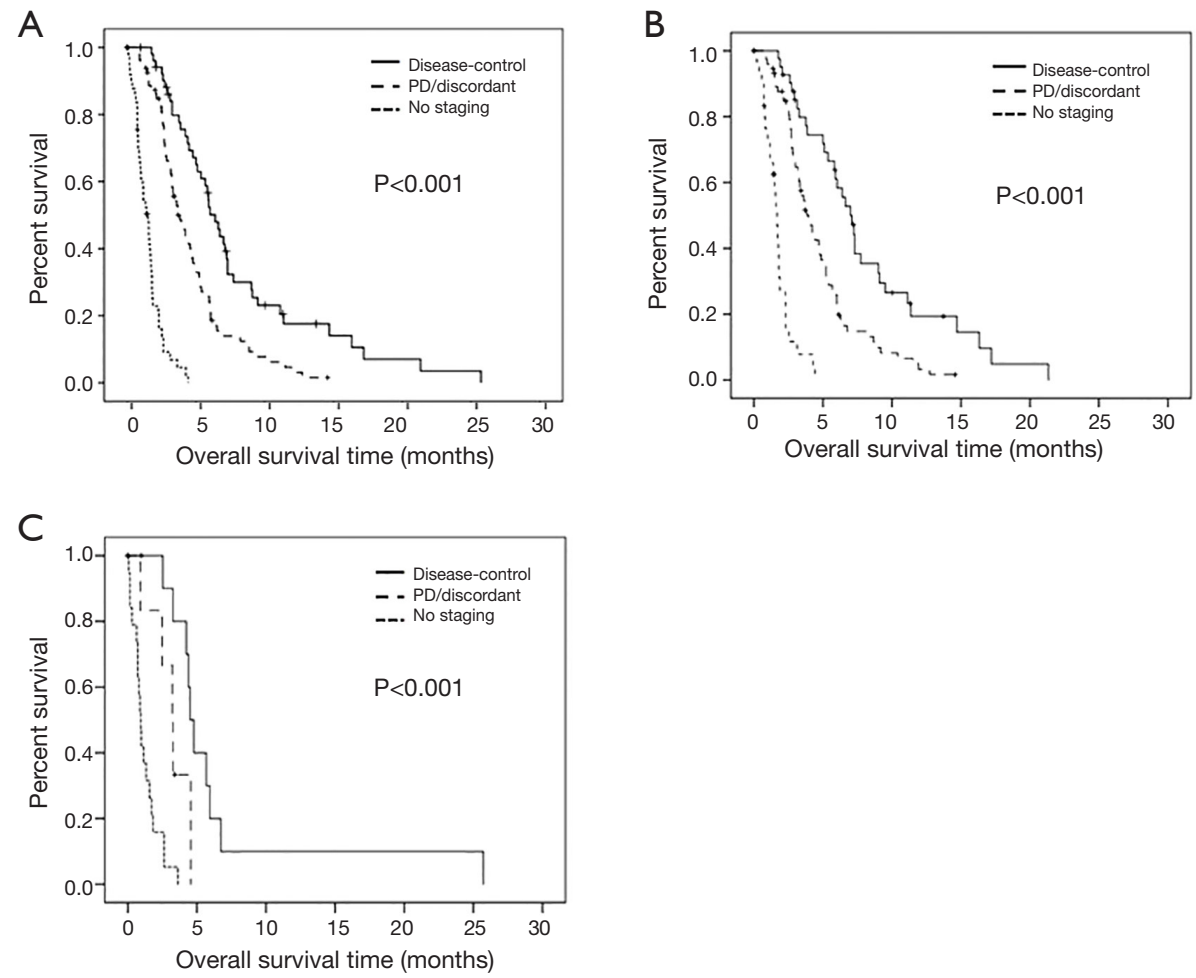

Figure S1 Kaplan Meier overall survival of paclitaxel-treated patients in this study, stratification according to response under paclitaxel therapy for (A) all paclitaxel-treated patients (6.4 vs. 3.8 vs. 1.5 months for DC vs. $\mathrm{PD} /$ discordant response $v s$. no staging; $\mathrm{P}<0.001)$, (B) paclitaxel-treated patients with ECOG PS 0-1 (7 vs. 3.9 vs. 1.7 months for DC vs. PD/discordant response vs. no staging, $\mathrm{P}<0.001 ;$ OS was 3.8 months for the entire ECOG PS 0-1 population, Figure 3B) and (C) paclitaxel-treated patients with ECOG PS 2 (4.5 vs. 3.2 vs. 0.9 months for $\mathrm{DC} v s$. $\mathrm{PD} /$ discordant response vs. no staging, $\mathrm{P}<0.001$; $\mathrm{DC} v s$. $\mathrm{PD} /$ discordant: $\mathrm{P}=0.039$; OS was 2.5 months for the entire $\mathrm{ECOG} \mathrm{PS} 2$ population, Figure 3B).

Table S1 Toxicities during paclitaxel therapy in ECOG PS 2 vs. ECOG PS 0-1 patients

\begin{tabular}{|c|c|c|c|c|c|c|}
\hline \multirow{2}{*}{ Toxicities $^{\S}$} & \multicolumn{3}{|c|}{ All grades, $\mathrm{n}$ [\%] } & \multicolumn{3}{|c|}{ CTC grade $3 / 4, n$ [\%] } \\
\hline & ECOG PS 2 & ECOG PS 0-1 & P value ${ }^{+}$ & ECOG PS 2 & ECOG PS 0-1 & $P$ value $^{+}$ \\
\hline Any toxicity & 22 [63] & 86 [62] & 0.91 & $5[14]$ & $23[16.5]$ & 0.74 \\
\hline Fatigue & $11[31]$ & 35 [25] & 0.56 & 3 [9] & $6[4]$ & 0.31 \\
\hline Polyneuropathy & $6[17]$ & 26 [13] & 0.83 & 0 & $3[2]$ & 0.38 \\
\hline Arthralgia/Myalgia & $6[17]$ & $22[11]$ & 0.85 & 0 & $3[2]$ & 0.38 \\
\hline Any hematotoxicity & $5[14]$ & $22[11]$ & 0.82 & $1[3]$ & $9[6]$ & 0.41 \\
\hline Leukocytopenia & $3[9]$ & $9[6]$ & 0.66 & $1[3]$ & $6[4]$ & 0.67 \\
\hline Anemia & $1[3]$ & $10[7]$ & 0.35 & 0 & $1[0.7]$ & 0.61 \\
\hline Thrombocytopenia & $1[3]$ & $4[3]$ & 0.99 & 0 & $2[1]$ & 0.48 \\
\hline Pancytopenia & 0 & $2[1]$ & 0.48 & 0 & $1[0.7]$ & 0.61 \\
\hline Nausea/vomiting & $4[11]$ & $21[15]$ & 0.56 & $2[6]$ & 4 [3] & 0.41 \\
\hline Hepatotoxicity & $1[3]$ & $6[4]$ & 0.67 & 0 & $2[1]$ & 0.48 \\
\hline Allergic reaction & $2[6]$ & $4[3]$ & 0.41 & 0 & $1[0.7]$ & 0.61 \\
\hline
\end{tabular}

§, data available for $n=35$ ECOG PS 2 patients and $n=139$ ECOG PS $0-1$ patients; ${ }^{+}$, comparison of toxicity rates between ECOG PS 2 patients and ECOG PS 0-1 patients with Chi-Square. 
Table S2 Response according to dose of paclitaxel therapy

\begin{tabular}{lcc}
\hline Response depending on dose reduction & $\mathrm{n}$ & $\%$ \\
\hline No dose reduction & 134 & 100 \\
PR & 25 & 19 \\
SD & 18 & 13 \\
PD & 55 & 41 \\
Discordant & 4 & 3 \\
Unknown & 32 & 24 \\
With dose reduction & 51 & 100 \\
PR & 6 & 12 \\
SD & 3 & 6 \\
PD & 19 & 37 \\
Discordant & 4 & 8 \\
Unknown & 19 & 37 \\
\hline
\end{tabular}

Table S3 Efficacy of paclitaxel therapy in patients with ECOG PS 2

\begin{tabular}{|c|c|c|c|c|c|}
\hline Best response in patients with ECOG PS 2, n [\%] & $\begin{array}{l}\text { 2nd line } \\
\qquad(n=3)\end{array}$ & $\begin{array}{c}\text { 3rd line } \\
(n=16)\end{array}$ & $\begin{array}{c}\text { 4th line } \\
(n=13)\end{array}$ & $\begin{array}{l}\text { 5th line } \\
(n=6)\end{array}$ & Overall $(n=38)$ \\
\hline DCR & $2[67]$ & $4[25]$ & 3 [23] & $2[33]$ & $11[29]$ \\
\hline PR & $1[33]$ & $2[12.5]$ & $2[15]$ & $1[17]$ & $6[16]$ \\
\hline SD & $1[33]$ & $2[12.5]$ & $1[8]$ & $1[17]$ & $5[13]$ \\
\hline PD & 0 & $1[6]$ & 3 [23] & 0 & $4[10.5]$ \\
\hline Discordant & 0 & $1[6]$ & $1[8]$ & 0 & $2[5]$ \\
\hline Unknown (no restaging performed) ${ }^{*}$ & 1 [33] & 10 [63.5] & $6[46]$ & $4[67]$ & 21 [55] \\
\hline
\end{tabular}

*, due to rapid clinical deterioration. 